
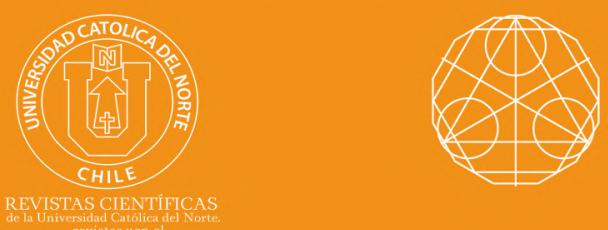

\title{
Radius problem for the class of analytic functions based on Ruscheweyh derivative
}

\author{
S. Mohapatra* iD orcid.org/0000-0002-4029-3392

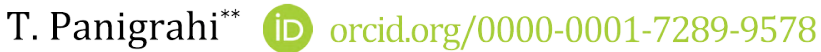

*KIIT (Deemed to be University), Dept. of Mathematics, Sch. of Applied Sciences, Bhubaneswa, OR, India susanta.kmohapatra1978@gmail.com

${ }^{*}$ KIIT (Deemed to be University), Dept. of Mathematics, Sch. of Applied Sciences, Bhubaneswar, QR, India. $\checkmark$ trailokyap6@gmail.com

\section{Received: May 2018 | Accepted: March 2019}

\section{Abstract:}

Let $\mathcal{A}$ be the class of analytic functions $f(z)$ with the normalized condition $f(0)=f^{\prime}(0)-1=0$ in the open unit disk $\mathbf{U}$. By making use of Ruscheweyh derivative operator, a new subclass $\mathcal{A}\left(\beta_{1}, \beta_{2}, \beta_{3}, \beta_{4} ; \lambda\right)$ of $f(z) \in \mathcal{A}$ satisfying the inequality

$\left|\beta_{1} z\left(\frac{D^{2} f(z)}{z}\right)^{\prime}+\beta_{2} z^{2}\left(\frac{D^{2} f(z)}{z}\right)^{\prime \prime}+\beta_{3} z^{3}\left(\frac{D^{2} f(z)}{z}\right)^{\prime \prime \prime}+\beta_{4} z^{4}\left(\frac{D^{2} f(z)}{z}\right)^{\prime \prime \prime \prime}\right| \leq$ $\lambda$,

for some complex numbers $\beta_{1}, \beta_{2}, \beta_{3}, \beta_{4}$ and for some real $\lambda>0$ is introduced. The object of the present paper is to obtain some properties of the function class $\mathcal{A}\left(\beta_{1}, \beta_{2}, \beta_{3}, \beta_{4} ; \lambda\right)$. Also the radius problems of $\frac{1}{\delta} f(\delta z) \in \mathcal{A}\left(\beta_{1}, \beta_{2}, \beta_{3}, \beta_{4} ; \lambda\right)$ where $f(z)$ satisfies the condition $\Re\left\{\frac{D^{2} f(z)}{z}\right\}>\alpha$ is considered.

Keywords: Analytic function; Univalent function; Ruscheweyh derivative; Cauchy-Schwarz inequality; Radius problema; Hölder inequality.

Mathematics Subject Classification (2010): 30C45, 30C50.

\section{Cite this article as (IEEE citation style):}

S. Mohapatra and T. Panigrahi, "Radius problem for the class of analytic functions based on Ruscheweyh derivative", Proyecciones (Antofagasta, On line), vol. 38, no. 3, pp. 541-556, Aug. 2019, doi: 10.22199/issn.0717-6279-2019-03-0034. [Accessed dd-mm-yyyy].

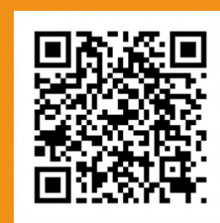

Article copyright: (C) 2019 Sushanta Kumar Mohapatra and Trailokya Panigrahi. This is an open access article distributed under the terms of the Creative Commons Licence, which permits unrestricted use and distribution provided the original author and source are credited. 


\section{Introduction and Motivation}

Let $\mathcal{A}_{0}$ be the class of functions $f(z)$ of the form:

$$
f(z)=a_{0}+a_{1} z+\sum_{n=2}^{\infty} a_{n} z^{n}
$$

which are analytic in the open unit disk

$\mathbf{U}:=\{\mathrm{z}: \mathrm{z} \in \mathbf{C}$ and $|z|<1\}$.

If $f(z) \in \mathcal{A}_{0}$ is given by (1.1), together with following normalization: $a_{0}=0$ and $a_{1}=1$, then we say that $f(z) \in \mathcal{A}$. Let $\mathcal{S}$ be the subclass of all functions in $\mathcal{A}$, which are univalent in $\mathbf{U}$.

For $f \in \mathcal{A}, \quad k \in \mathbf{N}_{0}:=\mathbf{N} \cup\{0\}$, Ruscheweyh [3] defined the operator $D^{k}: \mathcal{A} \longrightarrow \mathcal{A}$ by

$$
\begin{gathered}
D^{0} f(z)=f(z) \\
D^{1} f(z)=z f^{\prime}(z),
\end{gathered}
$$

$$
(k+1) D^{k+1} f(z)=z\left(D^{k} f(z)\right)^{\prime}+k\left(D^{k} f(z)\right) \quad(z \in \mathbf{U}) .
$$

Thus, if $f(z) \in \mathcal{A}, \quad f(z)=z+\sum_{n=2}^{\infty} a_{n} z^{n}$, then (see [5])

$$
D^{k} f(z)=z+\sum_{n=2}^{\infty} \frac{(k+n-1) !}{k !(n-1) !} a_{n} z^{n} \quad(z \in \mathbf{U}) .
$$

The Ruscheweyh operator has gained much aclaim as a unifying factor in the study of many classes of functions in geometric function theory.

Let $\mathcal{M}(\alpha)$ denote the subclass of $\mathcal{A}$ consisting of all $f(z)$ which satisfy the inequality

$$
\Re\left\{\frac{D^{2} f(z)}{z}\right\}>\alpha \quad(z \in \mathbf{U}) .
$$

For complex parameters $\beta_{1}, \beta_{2}$ and some real $\lambda>0$, Uyanì et al.[6] introduced the class $\mathcal{A}\left(\beta_{1}, \beta_{2} ; \lambda\right)$, a subclass of $\mathcal{A}$ as

$$
\left|\beta_{1} z\left(\frac{f(z)}{z}\right)^{\prime}+\beta_{2} z^{2}\left(\frac{f(z)}{z}\right)^{\prime \prime}\right| \leq \lambda \quad(z \in \mathbf{U}),
$$


and discussed some of its properties. Later on in 2011, Uyanì and Owa [7] introduced the third complex parameter $\beta_{3}$ and redefined the above class as

$$
\left|\beta_{1} z\left(\frac{f(z)}{z}\right)^{\prime}+\beta_{2} z^{2}\left(\frac{f(z)}{z}\right)^{\prime \prime}+\beta_{3} z^{3}\left(\frac{f(z)}{z}\right)^{\prime \prime \prime}\right| \leq \lambda \quad(z \in \mathbf{U})
$$

and denoted the class by $\mathcal{A}\left(\beta_{1}, \beta_{2}, \beta_{3} ; \lambda\right)$.

Motivated by the aforementioned work (also; see $[1,2]$ ), we define the subclass of $\mathcal{A}$ as follows.

Definition 1.1. A function $f(z) \in \mathcal{A}$ is said to be in the class $\mathcal{A}\left(\beta_{1}, \beta_{2}, \beta_{3}, \beta_{4} ; \lambda\right)$ if it satisfies the inequality

$$
\left|\beta_{1} z\left(\frac{D^{2} f(z)}{z}\right)^{\prime}+\beta_{2} z^{2}\left(\frac{D^{2} f(z)}{z}\right)^{\prime \prime}+\beta_{3} z^{3}\left(\frac{D^{2} f(z)}{z}\right)^{\prime \prime \prime}+\beta_{4} z^{4}\left(\frac{D^{2} f(z)}{z}\right)^{\prime \prime \prime \prime}\right| \leq \lambda,
$$

for some complex parameters $\beta_{1}, \beta_{2}, \beta_{3}, \beta_{4}$ and for some real number $\lambda>0$.

Example 1.2. Let us consider a function $f_{\gamma}(z)$ defined by

$$
\begin{gathered}
f_{\gamma}(z)=z(1+z)^{\gamma} \quad(\gamma \in \mathbf{R}) \\
=z+\sum_{n=2}^{\infty}\left(\begin{array}{c}
\gamma \\
n-1
\end{array}\right) z^{n}
\end{gathered}
$$

where

$$
\left(\begin{array}{c}
\gamma \\
n-1
\end{array}\right)=\frac{\gamma(\gamma-1)(\gamma-2) \ldots(\gamma-n+2)}{(n-1) !}(n \geq 2) .
$$

Then we have

$$
D^{2} f_{\gamma}(z)=z+\sum_{n=2}^{\infty} \frac{n(n+1)}{2}\left(\begin{array}{l}
\gamma \\
n-1
\end{array}\right) z^{n} .
$$

Therefore, it follows from (1.8) that

$$
\begin{aligned}
& \left|\beta_{1} z\left(\frac{D^{2} f_{\gamma}(z)}{z}\right)^{\prime}+\beta_{2} z^{2}\left(\frac{D^{2} f_{\gamma}(z)}{z}\right)^{\prime \prime}+\beta_{3} z^{3}\left(\frac{D^{2} f_{\gamma}(z)}{z}\right)^{\prime \prime \prime}+\beta_{4} z^{4}\left(\frac{D^{2} f_{\gamma}(z)}{z}\right)^{\prime \prime \prime \prime}\right| \\
& =\mid \sum_{n=2}^{\infty} \frac{n(n+1)}{2}\left(\begin{array}{c}
\gamma \\
n-1
\end{array}\right)
\end{aligned}
$$




$$
\left[\beta_{1}+(n-2) \beta_{2}+(n-2)(n-3) \beta_{3}+(n-2)(n-3)(n-4) \beta_{4}\right] z^{n-1} \mid .
$$

If $\gamma=1$, then from (1.9) we have

$$
\begin{aligned}
& \left|\beta_{1} z\left(\frac{D^{2} f_{1}(z)}{z}\right)^{\prime}+\beta_{2} z^{2}\left(\frac{D^{2} f_{1}(z)}{z}\right)^{\prime \prime}+\beta_{3} z^{3}\left(\frac{D^{2} f_{1}(z)}{z}\right)^{\prime \prime \prime}+\beta_{4} z^{4}\left(\frac{D^{2} f_{1}(z)}{z}\right)^{\prime \prime \prime \prime}\right| \\
& =\left|3 \beta_{1} z\right| \leq 3\left|\beta_{1}\right| .
\end{aligned}
$$

This implies that $f_{1}(z) \in \mathcal{A}\left(\beta_{1}, \beta_{2}, \beta_{3}, \beta_{4} ; \lambda\right)$ for $\lambda \geq 3\left|\beta_{1}\right|>0$.

If $\gamma=2$, then (1.9) yields

$$
\begin{aligned}
& \left|\beta_{1} z\left(\frac{D^{2} f_{2}(z)}{z}\right)^{\prime}+\beta_{2} z^{2}\left(\frac{D^{2} f_{2}(z)}{z}\right)^{\prime \prime}+\beta_{3} z^{3}\left(\frac{D^{2} f_{2}(z)}{z}\right)^{\prime \prime \prime}+\beta_{4} z^{4}\left(\frac{D^{2} f_{2}(z)}{z}\right)^{\prime \prime \prime \prime}\right| \\
& =\left|6 \beta_{1} z+12\left(\beta_{1}+\beta_{2}\right) z^{2}\right| \leq 18\left|\beta_{1}\right|+12\left|\beta_{2}\right|=6\left(3\left|\beta_{1}\right|+2\left|\beta_{2}\right|\right),
\end{aligned}
$$

which shows that $f_{2}(z) \in \mathcal{A}\left(\beta_{1}, \beta_{2}, \beta_{3}, \beta_{4}, \lambda\right)$ for $\lambda \geq 6\left(3\left|\beta_{1}\right|+2\left|\beta_{2}\right|\right)>0$.

Further, if $\gamma=3$, then from (1.9) we obtain

$$
\begin{aligned}
& \left|\beta_{1} z\left(\frac{D^{2} f_{3}(z)}{z}\right)^{\prime}+\beta_{2} z^{2}\left(\frac{D^{2} f_{3}(z)}{z}\right)^{\prime \prime}+\beta_{3} z^{3}\left(\frac{D^{2} f_{3}(z)}{z}\right)^{\prime \prime \prime}+\beta_{4} z^{4}\left(\frac{D^{2} f_{3}(z)}{z}\right)^{\prime \prime \prime \prime}\right| \\
& =\left|9 \beta_{1} z+36\left(\beta_{1}+\beta_{2}\right) z^{2}+30\left(\beta_{1}+2 \beta_{2}+2 \beta_{3}\right) z^{3}\right| \\
& \leq 75\left|\beta_{1}\right|+96\left|\beta_{2}\right|+60\left|\beta_{3}\right|=3\left[25\left|\beta_{1}\right|+32\left|\beta_{2}\right|+20\left|\beta_{3}\right|\right] .
\end{aligned}
$$

Thus $f_{3}(z) \in \mathcal{A}\left(\beta_{1}, \beta_{2}, \beta_{3}, \beta_{4} ; \lambda\right)$ for $\lambda \geq 3\left[25\left|\beta_{1}\right|+32\left|\beta_{2}\right|+20\left|\beta_{3}\right|\right]$.

Similarly, for $\gamma=4$, we have

$\left|\beta_{1} z\left(\frac{D^{2} f_{4}(z)}{z}\right)^{\prime}+\beta_{2} z^{2}\left(\frac{D^{2} f_{4}(z)}{z}\right)^{\prime \prime}+\beta_{3} z^{3}\left(\frac{D^{2} f_{4}(z)}{z}\right)^{\prime \prime \prime}+\beta_{4} z^{4}\left(\frac{D^{2} f_{4}(z)}{z}\right)^{\prime \prime \prime \prime}\right|$

$=\left|12 \beta_{1} z+72\left(\beta_{1}+\beta_{2}\right) z^{2}+120\left(\beta_{1}+2 \beta_{2}+2 \beta_{3}\right) z^{3}+60\left(\beta_{1}+3 \beta_{2}+6 \beta_{3}+6 \beta_{4}\right) z^{4}\right|$

$\leq 264\left|\beta_{1}\right|+492\left|\beta_{2}\right|+600\left|\beta_{3}\right|+360\left|\beta_{4}\right|=12\left[22\left|\beta_{1}\right|+41\left|\beta_{2}\right|+50\left|\beta_{3}\right|+30\left|\beta_{4}\right|\right]$.

Thus, $f_{4}(z) \in \mathcal{A}\left(\beta_{1}, \beta_{2}, \beta_{3}, \beta_{4} ; \lambda\right)$ for $\lambda \geq 12\left[22\left|\beta_{1}\right|+41\left|\beta_{2}\right|+50\left|\beta_{3}\right|+30\left|\beta_{4}\right|\right]$.

\section{Properties of the class $\mathcal{A}\left(\beta_{1}, \beta_{2}, \beta_{3}, \beta_{4} ; \lambda\right)$}

Our first result provides a sufficient condition for a function $f \in \mathcal{A}$ to be in the class $\mathcal{A}\left(\beta_{1}, \beta_{2}, \beta_{3}, \beta_{4} ; \lambda\right)$.

Theorem 2.1. If $f(z) \in \mathcal{A}$ satisfies

$$
\begin{gathered}
\sum_{n=2}^{\infty} \frac{n(n+1)}{2}(n-1)\left[\left|\beta_{1}\right|+(n-2)\left|\beta_{2}\right|\right. \\
\left.+(n-2)(n-3)\left|\beta_{3}\right|+(n-2)(n-3)(n-4)\left|\beta_{4}\right|\right]\left|a_{n}\right| \leq \lambda,
\end{gathered}
$$


for some complex numbers $\beta_{1}, \beta_{2}, \beta_{3}, \beta_{4}$ and for some real $\lambda>0$, then $f(z) \in \mathcal{A}\left(\beta_{1}, \beta_{2}, \beta_{3}, \beta_{4} ; \lambda\right)$.

Proof. Suppose that (2.1) holds true for some real $\lambda>0$. Then

$$
\begin{aligned}
& \left|\beta_{1} z\left(\frac{D^{2} f(z)}{z}\right)^{\prime}+\beta_{2} z^{2}\left(\frac{D^{2} f(z)}{z}\right)^{\prime \prime}+\beta_{3} z^{3}\left(\frac{D^{2} f(z)}{z}\right)^{\prime \prime \prime}+\beta_{4} z^{4}\left(\frac{D^{2} f(z)}{z}\right)^{\prime \prime \prime \prime}\right| \\
& =\mid \sum_{n=2}^{\infty} \frac{n(n+1)}{2}(n-1)\left[\beta_{1}+(n-2) \beta_{2}+(n-2)(n-3) \beta_{3}\right. \\
& \left.+(n-2)(n-3)(n-4) \beta_{4}\right] a_{n} z^{n-1} \mid \\
& \leq \sum_{n=2}^{\infty} \frac{n(n+1)}{2}(n-1)\left[\left|\beta_{1}\right|+(n-2)\left|\beta_{2}\right|\right. \\
& \left.+(n-2)(n-3)\left|\beta_{3}\right|+(n-2)(n-3)(n-4)\left|\beta_{4}\right|\right]\left|a_{n}\right||z|^{n-1} \\
& <\sum_{n=2}^{\infty} \frac{n(n+1)}{2}(n-1)\left[\left|\beta_{1}\right|+(n-2)\left|\beta_{2}\right|\right. \\
& \left.+(n-2)(n-3)\left|\beta_{3}\right|+(n-2)(n-3)(n-4)\left|\beta_{4}\right|\right] \mid a_{n} \\
& \leq \lambda,
\end{aligned}
$$

by virtue of (2.1). This shows that

$$
f(z) \in A\left(\beta_{1}, \beta_{2}, \beta_{3}, \beta_{4} ; \lambda\right) .
$$

Thus, the proof of Theorem 2.1 is completed.

The next theorem gives the necessary condition for the function $f \in \mathcal{A}$ to be in the class $\mathcal{A}\left(\beta_{1}, \beta_{2}, \beta_{3}, \beta_{4} ; \lambda\right)$.

Theorem 2.2. If $f(z) \in \mathcal{A}\left(\beta_{1}, \beta_{2}, \beta_{3}, \beta_{4} ; \lambda\right)$ with $\arg \beta_{1}=\arg \beta_{2}=\arg \beta_{3}=$ $\arg \beta_{4}=\psi$ and $a_{n}=\left|a_{n}\right| e^{i((n-1) \beta-\psi)}(n=2,3,4, \cdots)$, then

$$
\begin{gathered}
\sum_{n=2}^{\infty} \frac{n(n+1)}{2}(n-1) \\
{\left[\left|\beta_{1}\right|+(n-2)\left|\beta_{2}\right|+(n-2)(n-3)\left|\beta_{3}\right|+(n-2)(n-3)(n-4)\left|\beta_{4}\right|\right]\left|a_{n}\right| \leq \lambda .}
\end{gathered}
$$

Proof. If $f(z) \in \mathcal{A}\left(\beta_{1}, \beta_{2}, \beta_{3}, \beta_{4} ; \lambda\right)$ with $\arg \beta_{1}=\arg \beta_{2}=\arg \beta_{3}=$ $\arg \beta_{4}=\psi$ and $a_{n}=\left|a_{n}\right| e^{i((n-1) \beta-\psi)}$, then we have

$$
\begin{aligned}
& \left|\beta_{1} z\left(\frac{D^{2} f(z)}{z}\right)^{\prime}+\beta_{2} z^{2}\left(\frac{D^{2} f(z)}{z}\right)^{\prime \prime}+\beta_{3} z^{3}\left(\frac{D^{2} f(z)}{z}\right)^{\prime \prime \prime}+\beta_{4} z^{4}\left(\frac{D^{2} f(z)}{z}\right)^{\prime \prime \prime \prime}\right| \\
& =\mid \sum_{n=2}^{\infty} \frac{n(n+1)}{2}(n-1)\left[\beta_{1}+(n-2) \beta_{2}+(n-2)(n-3) \beta_{3}\right. \\
& \left.+(n-2)(n-3)(n-4) \beta_{4}\right] a_{n} z^{n-1} \mid \\
& =\mid \sum_{n=2}^{\infty} \frac{n(n+1)}{2}(n-1)\left[\left|\beta_{1}\right|+(n-2)\left|\beta_{2}\right|+(n-2)(n-3)\left|\beta_{3}\right|\right. \\
& \left.\quad+(n-2)(n-3)(n-4)\left|\beta_{4}\right|\right]\left|a_{n}\right| e^{i(n-1) \beta-\psi)} z^{n-1} \mid \leq \lambda,
\end{aligned}
$$


for all $z \in \mathbf{U}$. Let us consider a point $z \in \mathbf{U}$ such that $z=|z| e^{-i \beta}$. Then it follows from (2.3) that

$$
\begin{aligned}
& \left|\beta_{1} z\left(\frac{D^{2} f(z)}{z}\right)^{\prime}+\beta_{2} z^{2}\left(\frac{D^{2} f(z)}{z}\right)^{\prime \prime}+\beta_{3} z^{3}\left(\frac{D^{2} f(z)}{z}\right)^{\prime \prime \prime}+\beta_{4} z^{4}\left(\frac{D^{2} f(z)}{z}\right)^{\prime \prime \prime \prime}\right| \\
& =\sum_{n=2}^{\infty} \frac{n(n+1)}{2}(n-1)\left[\left|\beta_{1}\right|+(n-2)\left|\beta_{2}\right|\right. \\
& +(n-2)(n-3)\left|\beta_{3}\right|+(n-2)(n-3)(n-4)\left|\beta_{4}\right|\left|a_{n}\right||z|^{n-1} \leq \lambda .
\end{aligned}
$$

Letting $|z| \longrightarrow 1^{-}$, we obtain

$$
\begin{aligned}
& \sum_{n=2}^{\infty} \frac{n(n+1)}{2}(n-1)\left[\left|\beta_{1}\right|+(n-2)\left|\beta_{2}\right|+(n-2)(n-3)\left|\beta_{3}\right|\right. \\
& \left.\quad+(\mathrm{n}-2)(\mathrm{n}-3)(\mathrm{n}-4)\left|\beta_{4}\right|\right]\left|a_{n}\right| \leq \lambda .
\end{aligned}
$$

Thus, the proof of Theorem 2.2 is completed.

Corollary 2.3. If $f(z) \in \mathcal{A}\left(\beta_{1}, \beta_{2}, \beta_{3}, \beta_{4}, \lambda\right)$ with $\arg \beta_{1}=\arg \beta_{2}=\arg \beta_{3}=$ $\arg \beta_{4}=\psi$ and $a_{n}=\left|a_{n}\right| e^{i((n-1) \beta-\psi)} \quad(n=2,3, \cdots)$ then

$$
\begin{aligned}
& \left|a_{n}\right| \leq \frac{2 \lambda}{n(n+1)(n-1)\left[\left|\beta_{1}\right|+(n-2)\left|\beta_{2}\right|+(n-2)(n-3)\left|\beta_{3}\right|+(n-2)(n-3)(n-4)\left|\beta_{4}\right|\right]} \\
& \quad(n=2,3,4, \ldots) .
\end{aligned}
$$

A distortion property for the function $f$ in the class $A\left(\beta_{1}, \beta_{2}, \beta_{3}, \beta_{4} ; \lambda\right)$ is considered in the following corollary.

Corollary 2.4. If $f(z) \in \mathcal{A}\left(\beta_{1}, \beta_{2}, \beta_{3}, \beta_{4} ; \lambda\right)$ with $\arg \beta_{1}=\arg \beta_{2}=\arg \beta_{3}=$ $\arg \beta_{4}=\psi$ and $a_{n}=\left|a_{n}\right| e^{i((n-1) \beta-\psi)} \quad(n=2,3,4, \cdots)$, then we have

$$
|z|-\sum_{n=2}^{\infty}\left|a_{n}\right||z|^{n}-C_{j}|z|^{j+1} \leq|f(z)| \leq|z|+\sum_{n=2}^{\infty}\left|a_{n}\right||z|^{n}+C_{j}|z|^{j+1},
$$

with

$$
C_{j}=\frac{\lambda-\sum_{n=2}^{\infty} \frac{n(n+1)}{2}(n-1)\left[\left|\beta_{1}\right|+(n-2)\left|\beta_{2}\right|+(n-2)(n-3)\left|\beta_{3}\right|+(n-2)(n-3)(n-4)\left|\beta_{4}\right|\right]\left|a_{n}\right|}{\frac{(j+1)(j+2)}{2} j\left[\left|\beta_{1}\right|+(j-1)\left|\beta_{2}\right|+(j-1)(j-2)\left|\beta_{3}\right|+(j-1)(j-2)(j-3)\left|\beta_{4}\right|\right]}
$$


$1-\sum_{n=2}^{j} n\left|a_{n}\right||z|^{n-1}-Q_{j}|z|^{j} \leq\left|f^{\prime}(z)\right| \leq 1+\sum_{n=2}^{j} n\left|a_{n}\right||z|^{n-1}+Q_{j}|z|^{j} \quad(z \in \mathbf{U})$

with

$Q_{j}=\frac{2\left[\lambda-\sum_{n=2}^{\infty} \frac{n(n+1)(n-1)}{2}\left\{\left|\beta_{1}\right|+(n-2)\left|\beta_{2}\right|+(n-2)(n-3)\left|\beta_{3}\right|+(n-2)(n-3)(n-4)\left|\beta_{4}\right|\right\}\left|a_{n}\right|\right]}{j(j+2)\left[\left|\beta_{1}\right|+(j-1)\left|\beta_{2}\right|+(j-1)(j-2)\left|\beta_{3}\right|+(j-1)(j-2)(j-3)\left|\beta_{4}\right|\right]}$.

Proof. Since $f(z) \in \mathcal{A}\left(\beta_{1}, \beta_{2}, \beta_{3}, \beta_{4} ; \lambda\right)$, hence it follows from Theorem 2.2 that

$$
\begin{aligned}
& \sum_{n=j+1}^{\infty} \frac{n(n+1)}{2}(n-1)\left[\left|\beta_{1}\right|+(n-2)\left|\beta_{2}\right|+(n-2)(n-3)\left|\beta_{3}\right|+(n-2)(n-3)(n-4)\left|\beta_{4}\right|\right]\left|a_{n}\right| \\
& \leq \lambda-\sum_{n=2}^{j} \frac{n(n+1)}{2}(n-1)\left[\left|\beta_{1}\right|+(n-2)\left|\beta_{2}\right|+(n-2)(n-3)\left|\beta_{3}\right|+(n-2)(n-3)(n-4)\left|\beta_{4}\right|\right]\left|a_{n}\right| .
\end{aligned}
$$

Further, we note that

$$
\begin{aligned}
& \frac{j(j+1)(j+2)}{2}\left[\left|\beta_{1}\right|+(j-1)\left|\beta_{2}\right|+(j-1)(j-2)\left|\beta_{3}\right|\right. \\
& +(j-1)(j-2)(j-3)\left|\beta_{4}\right| \sum_{n=j+1}^{\infty}\left|a_{n}\right| \\
& \leq \sum_{n=j+1}^{\infty} \frac{n(n+1)(n-1)}{2}\left[\left|\beta_{1}\right|++(n-2)\left|\beta_{2}\right|+(n-2)(n-3)\left|\beta_{3}\right|\right. \\
& \quad+(n-2)(n-3)(n-4)\left|\beta_{4}\right|\left|a_{n}\right| .
\end{aligned}
$$

It follows from (2.8) and (2.9) that

$$
\begin{aligned}
& \sum_{n=j+1}^{\infty}\left|a_{n}\right| \\
\leq & \frac{\lambda-\sum_{n=2}^{j} \frac{n(n+1)(n-1)}{2}\left[\left|\beta_{1}\right|+(n-2)\left|\beta_{2}\right|+(n-2)(n-3)\left|\beta_{3}\right|+(n-2)(n-3)(n-4)\left|\beta_{4}\right|\right]\left|a_{n}\right|}{\frac{j(j+1)(j+2)}{2}}\left[\left|\beta_{1}\right|+(j-1)\left|\beta_{2}\right|+(j-1)(j-2)\left|\beta_{3}\right|+(j-1)(j-2)(j-3)\left|\beta_{4}\right|\right] \\
= & C_{j},
\end{aligned}
$$

where $C_{j}$ is given in $(2.5)$.

Since $f(z)=z+\sum_{n=2}^{\infty} a_{n} z^{n}$, which implies

$$
|f(z)| \leq|z|+\sum_{n=2}^{j}\left|a_{n}\right||z|^{n}+\sum_{n=j+1}^{\infty}\left|a_{n}\right||z|^{n}
$$




$$
\leq|z|+\sum_{n=2}^{j}\left|a_{n}\right||z|^{n}+C_{j}|z|^{j+1}
$$

and

$$
\begin{aligned}
|f(z)| & \geq|z|-\sum_{n=2}^{j}\left|a_{n}\right||z|^{n}-\sum_{n=j+1}^{\infty}\left|a_{n}\right||z|^{n} \\
& \geq|z|-\sum_{n=2}^{j}\left|a_{n}\right||z|^{n}-C_{j}|z|^{j+1} .
\end{aligned}
$$

Thus, the assertion (2.4) follows from (2.10) and (2.11). Further, we note that

$$
\begin{aligned}
\frac{(j+2)}{2} j\left[\left|\beta_{1}\right|+\right. & \left.(j-1)\left|\beta_{2}\right|+(j-1)(j-2)\left|\beta_{3}\right|+(j-1)(j-2)(j-3)\left|\beta_{4}\right|\right] \sum_{n=j+1}^{\infty} n\left|a_{n}\right| \\
& \leq \sum_{n=j+1}^{\infty} \frac{n(n+1)}{2}(n-1)\left[\left|\beta_{1}\right|+(n-2)\left|\beta_{2}\right|+(n-2)(n-3)\left|\beta_{3}\right|\right. \\
& \left.+(n-2)(n-3)(n-4)\left|\beta_{4}\right|\right]\left|a_{n}\right| \\
& \leq \lambda-\sum_{n=2}^{j} \frac{n(n+1)}{2}(n-1)\left[\left|\beta_{1}\right|+(n-2)\left|\beta_{2}\right|+(n-2)(n-3)\left|\beta_{3}\right|\right. \\
& \left.+(n-2)(n-3)(n-4)\left|\beta_{4}\right|\right]\left|a_{n}\right|,
\end{aligned}
$$

which yields

$$
\begin{aligned}
& \sum_{n=j+1}^{\infty} n\left|a_{n}\right| \\
& \leq \frac{2\left[\lambda-\sum_{n=2}^{\infty} \frac{n(n+1)}{2}(n-1)\left\{\left|\beta_{1}\right|+(n-2)\left|\beta_{2}\right|+(n-2)(n-3)\left|\beta_{3}\right|+(n-2)(n-3)(n-4)\left|\beta_{4}\right|\right\}\left|a_{n}\right|\right]}{\left.(j+2) j|| \beta_{1}|+(j-1)| \beta_{2}|+(j-1)(j-2)| \beta_{3}|+(j-1)(j-2)(j-3)| \beta_{4} \mid\right]} \\
& =Q_{j}
\end{aligned}
$$

where $Q_{j}$ is given by (2.7). Now

$$
\left|f^{\prime}(z)\right| \leq 1+\sum_{n=2}^{j} n\left|a_{n}\right||z|^{n-1}+\sum_{n=j+1}^{\infty} n\left|a_{n}\right||z|^{n-1} \leq 1+\sum_{n=2}^{j} n\left|a_{n}\right||z|^{n-1}+Q_{j}|z|^{j},
$$

and

$$
\begin{aligned}
\left|f^{\prime}(z)\right| \geq & 1-\sum_{n=2}^{j} n\left|a_{n}\right||z|^{n-1}-\sum_{n=j+1}^{\infty} n\left|a_{n}\right||z|^{n-1} \\
& \geq 1-\sum_{n=2}^{j} n\left|a_{n}\right||z|^{n-1}-Q_{j}|z|^{j}
\end{aligned}
$$

Combining (2.12) and (2.13) we obtain the inequality (2.6). This completes the proof of Corollary 2.4. 


\section{Radius Problem for the class $M(\beta, \alpha)$}

Let $\mathcal{A}_{\beta}$ denote the subclass of $\mathcal{A}$ consisting of all functions $f(z)$ with $a_{n}=\left|a_{n}\right| e^{i((n-1) \beta+\pi)} \quad(n=2,3, \cdots)$. If $\beta=0$, then a function $f(z) \in \mathcal{A}_{\beta}$ becomes an analytic function with negative coefficients in $\mathbf{U}$ considered by Silverman [4].

For $f(z) \in \mathcal{A}_{\beta}$, define the class $\mathcal{M}(\beta, \alpha)$ by

$$
\mathcal{M}(\beta, \alpha)=\mathcal{A}_{\beta} \cap \mathcal{M}(\alpha) \quad(0 \leq \alpha<1) .
$$

To obtain the radius problem for the class $\mathcal{M}(\beta, \alpha)$, we need the following lemma.

Lemma 3.1. If $f(z) \in \mathcal{M}(\beta, \alpha)$ then

$$
\sum_{n=2}^{\infty} \frac{n(n+1)}{2}\left|a_{n}\right| \leq 1-\alpha \quad(0 \leq \alpha<1) .
$$

Proof. Let $f(z) \in \mathcal{M}(\beta, \alpha)$. Then we have

$$
\begin{aligned}
& \Re\left[\frac{D^{2} f(z)}{z}\right]=\Re\left[1+\sum_{n=2}^{\infty} \frac{n(n+1)}{2} a_{n} z^{n-1}\right] \\
& =\Re\left[1+\sum_{n=2}^{\infty} \frac{n(n+1)}{2}\left|a_{n}\right| e^{i((n-1) \beta+\pi)} z^{n-1}\right] \\
& =\Re\left[1-\sum_{n=2}^{\infty} \frac{n(n+1)}{2}\left|a_{n}\right| e^{i(n-1) \beta} z^{n-1}\right]>\alpha,
\end{aligned}
$$

for all $z \in \mathbf{U}$. Let us consider a point $z \in \mathbf{U}$ such that $z=|z| e^{-i \beta}$. Then we have

$$
1-\sum_{n=2}^{\infty} \frac{n(n+1)}{2}\left|a_{n}\right||z|^{n-1}>\alpha .
$$

Therefore, letting $|z| \longrightarrow 1^{-1}$, we obtain

$$
\sum_{n=2}^{\infty} \frac{n(n+1)}{2}\left|a_{n}\right| \leq 1-\alpha .
$$

Corollary 3.2. If $f(z) \in \mathcal{M}(\beta, \alpha)$, then

$$
\left|a_{n}\right| \leq \frac{2(1-\alpha)}{n(n+1)} \quad(n=2,3, \cdots) .
$$


Remark 3.3. By Lemma 3.1, we observe that if $f(z) \in \mathcal{M}(\beta, \alpha)$, then

$$
\sum_{n=2}^{\infty} \frac{(n-1)(n+1)}{2}\left|a_{n}\right| \leq \sum_{n=2}^{\infty} \frac{n(n+1)}{2}\left|a_{n}\right| \leq 1-\alpha
$$

Now, we derive the following:

Theorem 3.4. If $f(z) \in \mathcal{M}(\beta, \alpha)$ and $\delta \in \mathbf{C} \quad(0<|\delta|<1)$. Then the function $\frac{1}{\delta} f(\delta z) \in A\left(\beta_{1}, \beta_{2}, \beta_{3}, \beta_{4}, \lambda\right)$ for $0<|\delta| \leq\left|\delta_{0}(\lambda)\right|$ where $\left|\delta_{0}(\lambda)\right|$ is the smallest positive root of the equation

$$
\begin{aligned}
& \frac{\left|\beta_{1}\right||\delta|}{\left(1-|\delta|^{2}\right)^{\frac{5}{2}}} \sqrt{6\left(1+|\delta|^{2}\right)} \sqrt{(1-\alpha)}+\frac{2\left|\beta_{2}\right||\delta|^{2}}{\left(1-|\delta|^{2}\right)^{\frac{7}{2}}} \sqrt{9+57|\delta|^{2}+24|\delta|^{4}} \\
& \sqrt{1-\alpha-\frac{3}{2}\left|a_{2}\right|^{2}} \\
& +\frac{4\left|\beta_{3}\right||\delta|^{3}}{\left(1-|\delta|^{2}\right)^{\frac{9}{2}}} \sqrt{30+405|\delta|^{2}+675|\delta|^{4}+150|\delta|^{6}} \sqrt{1-\alpha-\frac{3}{2}\left|a_{2}\right|^{2}-4\left|a_{3}\right|^{2}} \\
& +\frac{12\left|\beta_{4}\right| \mid \delta \delta^{4}}{\left(1-|\delta|^{2}\right)^{\frac{11}{2}}} \sqrt{75+1695|\delta|^{2}+805|\delta|^{4}+4425|\delta|^{6}+1000|\delta|^{8}} \\
& \sqrt{1-\alpha-\frac{3}{2}\left|a_{2}\right|^{2}-4\left|a_{3}\right|^{2}-\frac{15}{2}\left|a_{4}\right|^{2}}=\lambda .
\end{aligned}
$$

Proof. For $f(z) \in \mathcal{M}(\beta, \alpha)$, we see that

$$
\frac{1}{\delta} f(\delta z)=z+\sum_{n=2}^{\infty} \delta^{n-1} a_{n} z^{n} \quad(0<|\delta|<1) .
$$

To show $\frac{1}{\delta} f(\delta z) \in A\left(\beta_{1}, \beta_{2}, \beta_{3}, \beta_{4} ; \lambda\right)$. In view of Theorem 2.1, it is sufficient to prove

$$
\left.(3.4)+(n-2)(n-3)\left|\beta_{3}\right|+(n-2)(n-3)(n-4)\left|\beta_{4}\right|\right]|\delta|^{n-1}\left|a_{n}\right| \leq \lambda .
$$

We note that

$$
\sum_{n=2}^{\infty} \frac{(n+1)(n-1)}{2}\left|a_{n}\right|^{2} \leq 1-\alpha .
$$

Applying Cauchy-Schwarz inequality to the left hand side of (3.4), we observe that

$$
\begin{aligned}
& \sum_{n=2}^{\infty} \frac{n(n+1)}{2}(n-1)\left[\left|\beta_{1}\right|+(n-2)\left|\beta_{2}\right|\right. \\
& \left.+(n-2)(n-3)\left|\beta_{3}\right|+(n-2)(n-3)(n-4)\left|\beta_{4}\right|\right]|\delta|^{n-1}\left|a_{n}\right|
\end{aligned}
$$




$$
\begin{aligned}
& \leq \frac{\left|\beta_{1}\right|}{|\delta|}\left(\sum_{n=2}^{\infty} \frac{n^{2}(n+1)(n-1)}{2}|\delta|^{2 n}\right)^{\frac{1}{2}}\left(\sum_{n=2}^{\infty} \frac{(n+1)(n-1)}{2}\left|a_{n}\right|^{2}\right)^{\frac{1}{2}} \\
& +\frac{\left|\beta_{2}\right|}{|\delta|}\left(\sum_{n=3}^{\infty} \frac{n^{2}(n+1)(n-1)(n-2)^{2}}{2}|\delta|^{2 n}\right)^{\frac{1}{2}}\left(\sum_{n=3}^{\infty} \frac{(n+1)(n-1)}{2}\left|a_{n}\right|^{2}\right)^{\frac{1}{2}} \\
& +\frac{\left|\beta_{3}\right|}{|\delta|}\left(\sum_{n=4}^{\infty} \frac{n^{2}(n+1)(n-1)(n-2)^{2}(n-3)^{2}}{2}|\delta|^{2 n}\right)^{\frac{1}{2}}\left(\sum_{n=4}^{\infty} \frac{(n+1)(n-1)}{2}\left|a_{n}\right|^{2}\right)^{\frac{1}{2}} \\
& +\frac{\left|\beta_{4}\right|}{|\delta|}\left(\sum_{n=5}^{\infty} \frac{n^{2}(n+1)(n-1)(n-2)^{2}(n-3)^{2}(n-4)^{2}}{2}|\delta|^{2 n}\right)^{\frac{1}{2}}\left(\sum_{n=5}^{\infty} \frac{(n+1)(n-1)}{2}\left|a_{n}\right|^{2}\right)^{\frac{1}{2}} \\
& \leq \frac{\left|\beta_{1}\right|}{\delta \mid}\left(\sum_{n=2}^{\infty} \frac{n^{2}(n+1)(n-1)}{2}|\delta|^{2 n}\right)^{\frac{1}{2}} \sqrt{1-\alpha} \\
& +\frac{\left|\beta_{2}\right|}{|\delta|}\left(\sum_{n=3}^{\infty} \frac{n^{2}(n+1)(n-1)(n-2)^{2}}{2}|\delta|^{2 n}\right)^{\frac{1}{2}} \sqrt{1-\alpha-\frac{3}{2}\left|a_{2}\right|^{2}} \\
& +\frac{\left|\beta_{3}\right|}{|\delta|}\left(\sum_{n=4}^{\infty} \frac{n^{2}(n+1)(n-1)(n-2)^{2}(n-3)^{2}}{2}|\delta|^{2 n}\right)^{\frac{1}{2}} \sqrt{1-\alpha-\frac{3}{2}\left|a_{2}\right|^{2}-4\left|a_{3}\right|^{2}} \\
& +\frac{\left|\beta_{4}\right|}{|\delta|}\left(\sum_{n=5}^{\infty} \frac{n^{2}(n+1)(n-1)(n-2)^{2}(n-3)^{2}(n-4)^{2}}{2}|\delta|^{2 n}\right)^{\frac{1}{2}} \\
& \sqrt{1-\alpha-\frac{3}{2}\left|a_{2}\right|^{2}-4\left|a_{3}\right|^{2}-\frac{15}{2}\left|a_{4}\right|^{2}} .
\end{aligned}
$$

Making use of

$$
\sum_{n=2}^{\infty} x^{n}=\frac{x^{2}}{1-x} \quad(|x|<1)
$$

we have

$$
\sum_{n=2}^{\infty} n x^{n+1}=x^{2}\left(\sum_{n=2}^{\infty} x^{n}\right)^{\prime}=x^{2}\left(\frac{x^{2}}{1-x}\right)=\frac{2 x^{4}-x^{5}}{(1-x)^{2}} .
$$

Thus

$$
\begin{gathered}
\frac{1}{2} \sum_{n=2}^{\infty} n^{2}(n+1)(n-1) x^{n}=\frac{x^{2}}{2}\left(\sum_{n=2}^{\infty} n x^{n+1}\right)^{\prime \prime \prime} \\
=\frac{6 x^{2}(1+x)}{(1-x)^{5}}
\end{gathered}
$$

Since

$$
\sum_{n=3}^{\infty}(n-2) x^{n}=\frac{x^{3}}{(1-x)^{2}}
$$

we have

$$
\sum_{n=3}^{\infty} n(n-2) x^{n+1}=x^{2}\left(\sum_{n=3}^{\infty}(n-2) x^{n}\right)^{\prime}=\frac{3 x^{4}-x^{5}}{(1-x)^{3}} .
$$


Therefore

$$
\begin{gathered}
\sum_{n=3}^{\infty} \frac{n^{2}(n+1)(n-1)(n-2)^{2}}{2} x^{n}=\frac{x^{3}}{2}\left(\sum_{n=3}^{\infty} n(n-2) x^{n+1}\right)^{\prime \prime \prime \prime} \\
=\frac{36 x^{3}+228 x^{4}+96 x^{5}}{(1-x)^{7}}
\end{gathered}
$$

Again, we have

$$
\sum_{n=4}^{\infty}(n-2)(n-3) x^{n}=x^{4}\left(\sum_{n=4}^{\infty} x^{n-2}\right)^{\prime \prime}=\frac{2 x^{4}}{(1-x)^{3}},
$$

which implies

$$
\sum_{n=4}^{\infty} n(n-2)(n-3) x^{n+1}=x^{2}\left(\sum_{n=4}^{\infty}(n-2)(n-3) x^{n}\right)^{\prime}=\frac{8 x^{5}-2 x^{6}}{(1-x)^{4}} .
$$

Therefore

$$
\begin{gathered}
\sum_{n=4}^{\infty} \frac{n^{2}(n+1)(n-1)(n-2)^{2}(n-3)^{2}}{2} x^{n}=\frac{x^{4}}{2}\left(\sum_{n=4}^{\infty} n(n-2)(n-3) x^{n+1}\right)^{\prime \prime \prime \prime \prime \prime} \\
=\frac{480 x^{4}+6480 x^{5}+10800 x^{6}+2400 x^{7}}{(1-x)^{9}} .
\end{gathered}
$$

Furthermore, we have

$$
\sum_{n=5}^{\infty} n(n-2)(n-3)(n-4) x^{n+1}=x^{2}\left(\sum_{n=5}^{\infty}(n-2)(n-3)(n-4) x^{n}\right)^{\prime} .
$$

But

$$
\sum_{n=5}^{\infty}(n-2)(n-3)(n-4) x^{n}=x^{5}\left(\sum_{n=5}^{\infty} x^{n-2}\right)^{\prime \prime \prime}=\frac{6 x^{5}}{(1-x)^{4}} .
$$

Thus, we have

$$
\sum_{n=5}^{\infty} n(n-2)(n-3)(n-4) x^{n+1}=x^{2}\left(\frac{6 x^{5}}{(1-x)^{4}}\right)^{\prime}=\frac{30 x^{6}-6 x^{7}}{(1-x)^{5}} .
$$

This gives us

$$
\begin{aligned}
& \sum_{n=5}^{\infty} \frac{n^{2}(n+1)(n-1)(n-2)^{2}(n-3)^{2}(n-4)^{2}}{2} x^{n} \\
& =\frac{x^{5}}{2}\left(\sum_{n=5}^{\infty} n(n-2)(n-3)(n-4) x^{n+1}\right)^{\prime \prime \prime \prime \prime \prime \prime}
\end{aligned}
$$




$$
=\frac{10800 x^{5}+244080 x^{6}+835920 x^{7}+637200 x^{8}+144000 x^{9}}{(1-x)^{11}} .
$$

By using (3.6)- (3.9) with $|\delta|^{2}=x$ in (3.5), we obtain

$$
\begin{aligned}
& \sum_{n=2}^{\infty} \frac{n(n+1)}{2}(n-1)\left[\left|\beta_{1}\right|+(n-2)\left|\beta_{2}\right|+(n-2)(n-3)\left|\beta_{3}\right|\right. \\
& +(n-2)(n-3)(n-4)\left|\beta_{4}\right||\delta|^{n-1}\left|a_{n}\right| \\
& \leq \frac{\left|\beta_{1}\right||\delta|}{\left(1-|\delta|^{2}\right)^{\frac{5}{2}}} \sqrt{6\left(1+|\delta|^{2}\right)} \sqrt{(1-\alpha)}+\frac{2\left|\beta_{2}\right||\delta|^{2}}{\left(1-|\delta|^{2}\right)^{\frac{7}{2}}} \sqrt{9+57|\delta|^{2}+24|\delta|^{4}} \\
& \sqrt{1-\alpha-\frac{3}{2}\left|a_{2}\right|^{2}} \\
& +\frac{4\left|\beta_{3}\right||\delta|^{3}}{\left(1-|\delta|^{2}\right)^{\frac{9}{2}}} \sqrt{30+405|\delta|^{2}+675|\delta|^{4}+150|\delta|^{6}} \sqrt{1-\alpha-\frac{3}{2}\left|a_{2}\right|^{2}-4\left|a_{3}\right|^{2}} \\
& +\frac{12\left|\beta_{4}\right| \mid \delta \delta^{4}}{\left(1-|\delta|^{2}\right)^{\frac{11}{2}}} \sqrt{75+1695|\delta|^{2}+805|\delta|^{4}+4925|\delta|^{6}+1000|\delta|^{8}} \\
& \sqrt{1-\alpha-\frac{3}{2}\left|a_{2}\right|^{2}-4\left|a_{3}\right|^{2}-\frac{15}{2}\left|a_{4}\right|^{2}} .
\end{aligned}
$$

Let us consider the complex number $\delta(0<|\delta|<1)$ such that

$$
\begin{aligned}
& \frac{\left|\beta_{1}\right||\delta|}{\left(1-|\delta|^{2}\right)^{\frac{5}{2}}} \sqrt{6\left(1+|\delta|^{2}\right)} \sqrt{(1-\alpha)}+\frac{2\left|\beta_{2}\right||\delta|^{2}}{\left(1-|\delta|^{2}\right)^{\frac{7}{2}}} \sqrt{9+57|\delta|^{2}+24|\delta|^{4}} \\
& \sqrt{1-\alpha-\frac{3}{2}\left|a_{2}\right|^{2}} \\
& +\frac{4\left|\beta_{3}\right||\delta|^{3}}{\left(1-|\delta|^{2}\right)^{\frac{9}{2}}} \sqrt{30+405|\delta|^{2}+675|\delta|^{4}+150|\delta|^{6}} \sqrt{1-\alpha-\frac{3}{2}\left|a_{2}\right|^{2}-4\left|a_{3}\right|^{2}} \\
& +\frac{12\left|\beta_{4}\right||\delta|^{4}}{\left(1-|\delta|^{2}\right)^{\frac{11}{2}}} \sqrt{75+1695|\delta|^{2}+805|\delta|^{4}+4925|\delta|^{6}+1000|\delta|^{8}} \\
& \sqrt{1-\alpha-\frac{3}{2}\left|a_{2}\right|^{2}-4\left|a_{3}\right|^{2}-\frac{15}{2}\left|a_{4}\right|^{2}}=\lambda .
\end{aligned}
$$

If we define the function $h(|\delta|)$ by

$$
\begin{aligned}
& h(|\delta|)=\left|\beta_{1}\right||\delta|\left(1-|\delta|^{2}\right)^{3} \sqrt{6\left(1+|\delta|^{2}\right)} \sqrt{(1-\alpha)}+2\left|\beta_{2}\right||\delta|^{2}\left(1-|\delta|^{2}\right)^{2} \\
& \sqrt{9+57|\delta|^{2}+24|\delta|^{4}} \\
& \sqrt{1-\alpha-\frac{3}{2}\left|a_{2}\right|^{2}}+4\left|\beta_{3}\right||\delta|^{3}\left(1-|\delta|^{2}\right) \sqrt{30+405|\delta|^{2}+675|\delta|^{4}+150|\delta|^{6}} \\
& \sqrt{1-\alpha-\frac{3}{2}\left|a_{2}\right|^{2}-4\left|a_{3}\right|^{2}} \\
& +12\left|\beta_{4}\right||\delta|^{4} \sqrt{75+\left.1695\right|^{2}+\left.8\right|^{2}+85|\delta|^{4}+4425|\delta|^{6}+1000|\delta|^{8}} \\
& \sqrt{1-\alpha-\frac{3}{2}\left|a_{2}\right|^{2}-4\left|a_{3}\right|^{2}-\frac{15}{2}\left|a_{4}\right|^{2}}-\lambda\left(1-|\delta|^{2}\right)^{\frac{11}{2}},
\end{aligned}
$$

then we have $h(0)=-\lambda<0$ and 


$$
h(1)=60 \sqrt{3}\left|\beta_{4}\right| \sqrt{1-\alpha-\frac{3}{2}\left|a_{2}\right|^{2}-4\left|a_{3}\right|^{2}-\frac{15}{2}\left|a_{4}\right|^{2}} \geq 0 .
$$

This means that there exists some $\delta_{0}$ such that $h\left(\left|\delta_{0}\right|\right)=0 \quad\left(0<\left|\delta_{0}\right|<1\right)$. This completes the proof of the Theorem 3.1.

Remark 3.5. If we take $\delta=\frac{1}{2} e^{i \beta}$ in (3.3), then

$$
\begin{aligned}
& \lambda=\frac{8}{9} \sqrt{10}\left|\beta_{1}\right| \sqrt{1-\alpha}+\frac{32 \sqrt{11}}{9 \sqrt{3}}\left|\beta_{2}\right| \sqrt{1-\alpha-\frac{3}{2}\left|a_{2}\right|^{2}} \\
& +\frac{800 \sqrt{2}}{27 \sqrt{3}}\left|\beta_{3}\right| \sqrt{1-\alpha-\frac{3}{2}\left|a_{2}\right|^{2}-4\left|a_{3}\right|^{2}}+\frac{64 \sqrt{39815}}{81 \sqrt{3}}\left|\beta_{4}\right| \\
& \sqrt{1-\alpha-\frac{3}{2}\left|a_{2}\right|^{2}-4\left|a_{3}\right|^{2}-\frac{15}{2}\left|a_{4}\right|^{2}} .
\end{aligned}
$$

If we consider $\lambda=1$ in (3.3) then we obtain

$$
\begin{aligned}
& \left|\beta_{1}\right||\delta|\left(1-|\delta|^{2}\right)^{3} \sqrt{6\left(1+|\delta|^{2}\right)} \sqrt{1-\alpha}+2\left|\beta_{2}\right||\delta|^{2}\left(1-|\delta|^{2}\right)^{2} \\
& \sqrt{9+57|\delta|^{2}+24|\delta|^{4}} \sqrt{1-\alpha-\frac{3}{2}\left|a_{2}\right|^{2}} \\
& +4\left|\beta_{3}\right||\delta|^{3}\left(1-|\delta|^{2}\right) \sqrt{30+405|\delta|^{2}+675|\delta|^{4}+150|\delta|^{6}} \\
& \sqrt{1-\alpha-\frac{3}{2}\left|a_{2}\right|^{2}-4\left|a_{3}\right|^{2}} \\
& +12\left|\beta_{4}\right||\delta|^{4} \sqrt{75+1695|\delta|^{2}+805|\delta|^{4}+4425|\delta|^{6}+1000|\delta|^{8}} \\
& \sqrt{1-\alpha-\frac{3}{2}\left|a_{2}\right|^{2}-4\left|a_{3}\right|^{2}-\frac{15}{2}\left|a_{4}\right|^{2}}-\left(1-|\delta|^{2}\right)^{\frac{11}{2}}=0 .
\end{aligned}
$$

\section{Open Problem}

For the proof of Theorem 3.1 we used Cauchy- Schwarz inequality given by

$$
\sum\left|a_{n}\right|\left|b_{n}\right| \leq\left(\sum\left|a_{n}\right|^{2}\right)^{\frac{1}{2}}\left(\sum\left|b_{n}\right|^{2}\right)^{\frac{1}{2}}
$$

We know that Hölder inequality i.e

$$
\sum\left|a_{n}\right|\left|b_{n}\right| \leq\left(\sum\left|a_{n}\right|^{p}\right)^{\frac{1}{p}}\left(\sum\left|b_{n}\right|^{q}\right)^{\frac{1}{q}} \quad\left(p>0, q>0 ; \frac{1}{p}+\frac{1}{q} \geq 1\right)
$$

is the generalization of Cauchy-Schwarz inequality. Therefore, if we find some application of Hölder inequality for the proof of Theorem 3.4 instead of Cauchy-Schwarz inequality, then we derive new result which is the generalization of Theorem 3.4. 


\section{Acknowledgment:}

The authors would like to thank to the editor and anonymous referees for their comments and suggestions which improve the contents of the manuscript. Further, the present investigation of the second-named author is supported by CSIR research project scheme no: 25(0278)/17/EMR-II, New Delhi, India.

\section{References}

[1] 0. Kwon, Y. Sim, N. Cho, and H. Srivastava, "Some radius problems related to a certain subclass of analytic functions", Acta Mathematica Sinica, English Series, vol. 30, no. 7, pp. 1133-1144, Jul. 2014, doi: 10.1007/s10114-014-3100-0.

[2] S. Maharana, J. Prajapat, and H. Srivastava, "The radius of convexity of partial sums of convex functions in one direction", Proceedings of the National Academy of Sciences, India Section A: Physical Sciences, vol. 87, no. 2, pp. 215-219, Feb. 2017, doi: 10.1007/s40010-017-0348-7.

[3] S. Ruscheweyh, "New criteria for univalent functions", Proceedings of the American Mathematical Society, vol. 49, no. 1, pp. 109-109, Jan. 1975, doi: 10.1090/S0002-9939-1975-0367176-1.

[4] H. Silverman, "Univalent functions with negative coefficients", Proceedings of the American Mathematical Society, vol. 51, no. 1, pp. 109-109, Jan. 1975, doi: 10.1090/S0002-9939-1975-0369678-0.

[5] H. Srivastava, N. Xu, and D. Yang, "Inclusion relations and convolution properties of a certain class of analytic functions associated with the Ruscheweyh derivatives", Journal of Mathematical Analysis and Applications, vol. 331, no. 1, pp. 686-700, Jul. 2007, doi: 10.1016/j.jmaa.2006.09.019.

[6] N. Uyanì, S. Owa, and E. Kadioğlu, "Some properties of functions associated with close-to-convex and starlike of order", Applied Mathematics and Computation, vol. 216, no. 2, pp. 381-387, Mar. 2010, doi: 10.1016/j.amc.2010.01.022.

[7] N. Uyanì and S. Owa, "New extensions for classes of analytic functions associated with close-to-convex and starlike of order $\alpha$ ", Mathematical and Computer Modelling, vol. 54, no. 1-2, pp. 359-366, Jul. 2011, doi: 10.1016/j.mcm.2011.02.020. 